\title{
Quantum interference in thermoelectric molecular junctions: A toy model perspective
}

\author{
Daijiro Nozaki, ${ }^{1, a)}$ Stas M. Avdoshenko, ${ }^{2}$ Hâldun Sevinçlit, ${ }^{3}$ and Gianaurelio Cuniberti ${ }^{1,4,5}$ \\ ${ }^{1}$ Institute for Materials Science and Max Bergmann Center of Biomaterials, TU Dresden, 01062 Dresden, \\ Germany \\ ${ }^{2}$ Department of Chemistry and Institute for Computational Engineering and Sciences, University of Texas at \\ Austin, 100 E. 24th St. A1590, Austin, Texas 78712, USA \\ ${ }^{3}$ Department of Materials Science and Engineering, Izmir Institute of Technology, Gulbahce Kampusu 35430 \\ Urla, Izmir, Turkey \\ ${ }^{4}$ Dresden Center for Computational Materials Science (DCCMS), TU Dresden, 01062 Dresden, Germany \\ ${ }^{5}$ Center for Advancing Electronics Dresden (cfAED), TU Dresden, 01062 Dresden, Germany
}

(Received 6 May 2014; accepted 7 August 2014; published online 21 August 2014)

\begin{abstract}
Quantum interference (QI) phenomena between electronic states in molecular circuits offer a new opportunity to design new types of molecular devices such as molecular sensors, interferometers, and thermoelectric devices. Controlling the QI effect is a key challenge for such applications. For the development of single molecular devices employing QI effects, a systematic study of the relationship between electronic structure and the quantum interference is needed. In order to uncover the essential topological requirements for the appearance of QI effects and the relationship between the QI-affected line shape of the transmission spectra and the electronic structures, we consider a homogeneous toy model where all on-site energies are identical and model four types of molecular junctions due to their topological connectivities. We systematically analyze their transmission spectra, density of states, and thermoelectric properties. Even without the degree of freedom for on-site energies an asymmetric Fano peak could be realized in the homogeneous systems with the cyclic configuration. We also calculate the thermoelectric properties of the model systems with and without fluctuation of on-site energies. Even under the fluctuation of the on-site energies, the finite thermoelectrics are preserved for the Fano resonance, thus cyclic configuration is promising for thermoelectric applications. This result also suggests the possibility to detect the cyclic configuration in the homogeneous systems and the presence of the QI features from thermoelectric measurements. (C) 2014 AIP Publishing LLC. [http://dx.doi.org/10.1063/1.4893475]
\end{abstract}

\section{INTRODUCTION}

Similar to classical interference, quantum interference (QI) is a phenomenon which is induced by the superposition of wave functions, or propagating wavefunctions in nanostructures such as open quantum systems. Historically, the effect of QI on transport has been broadly studied in the field of mesoscopic physics, quantum dots, ${ }^{1-22}$ and electron transfer systems. ${ }^{23-25}$ Recently, the effect of QI on electron transport in molecular junctions has been getting an increasing amount of attention due to its unique physics and its possible applications in various fields. ${ }^{26-47,50-67}$ The QI effect emerges as additional peaks or dips in transmission spectra. These features can improve a performance of molecular switches/sensors, ${ }^{28,56}$ and thermoelectric devices. ${ }^{36,39}$ Using the inverse of this process, the QI effect on the thermoelectric properties can also be used to infer the electronic structure of the systems by measuring the thermoelectric current.

Due to the ease of management and control of QI effects by electronic structure or molecular topologies, the scope of the QI effect ranges from fundamental studies up to applied science frontiers in sensors or interferometer applications. ${ }^{62}$

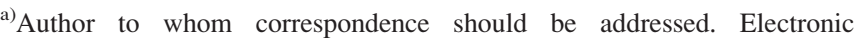
addresses: daijiro.nozaki@gmail.com and research@nano.tu-dresden.de
}

Continuous studies on the topic of QI have reported the distinct signatures of QI; in some cases there are almost symmetric negative peaks within the transmission range, the socalled anti-resonance (for instance, see Refs. 2, 27-29, 36, and 56), or it might be sharp asymmetric peaks - the socalled Fano resonance (for instance, see Refs. 30, 31, and 36). As a qualitative trend, the T-shaped ${ }^{1,31-33,36,37,65,66}$ or cyclic molecules ${ }^{34,35,41,43,54,67}$ tend to have QI-related features in their transmission spectra.

In the last few decades, several types of mechanisms to realize the QI effect in molecular devices have been proposed. $^{27,28,31,35-39,41-43,53,56,57}$ Associated with these mechanisms, many methods and techniques to understand and to predict the origin of the QI have been also studied. For the further development of single molecular devices employing QI effects, a systematic study of the relationship between electronic structure and the quantum interference is needed. In the rich parametric space of real organic molecules, it is difficult to highlight and understand the essential features responsible for the appearance of the QI effect in molecular junctions. Even a simplified picture, which is formed by onsite energies, transfer integrals, and the topology of the molecules itself, presents a rather complicated task to work with. Recently in addition to pure electronic QI, the QI due to the interaction with phonons ${ }^{47,68,69}$ has been also reported. The 
aim of this work is to uncover the essential topological requirements for the appearance of QI effects and to investigate its influence on thermoelectric transport. To eliminate the crowding and uncertainty in the richer parameter spaces, we restrict our interest to a purely electronic homogeneous minimal toy model where all on-site energies are equal.

In this study, we consider four different model configurations for the molecular conductors according to their electronic connectivities as shown in Fig. 1. From the qualitative trend in literature that the T-shaped and cyclic molecules tend to have QI-related features in their transmission spectra, we considered the T-shaped and cyclic configurations as shown in Figs. 1(b) and 1(c). Although our main targets are the T-shaped and cyclic configurations having QI features as shown in Figs. 1(b) and 1(c), we also considered the normal Breit-Wigner resonances (Figs. 1(a) and 1(d)) as references or for comparison. ${ }^{48,49}$ Then we systematically calculate electronic transmissions, density of states (DOS), and thermoelectric properties using a simple tight-binding model. The influence of the coupling strengths, phase shift, and thermal disorders on the transport are also examined using the toy model.

By analyzing a variety of topological orderings, we found that only the cyclically closed model junction gives an asymmetric transmission line shape, which leads to enhanced thermoelectric characteristics. Focusing on this topology in the final part of the manuscript, we selected a series of optimal conditions for higher thermoelectric response and conclude that QI tuning is important for sensoric and thermoelectric applications.

\section{THEORETICAL FRAMEWORK}

We calculated the conductance and the DOS of molecular junctions using the standard Landauer formula written in terms of Green's function formalisms. ${ }^{70,71}$ Figure 1 shows a (a) Tunneling

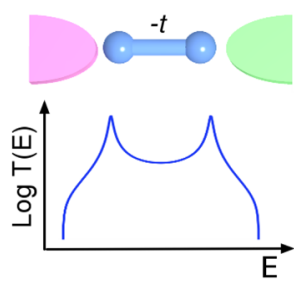

(c) Fano resonance

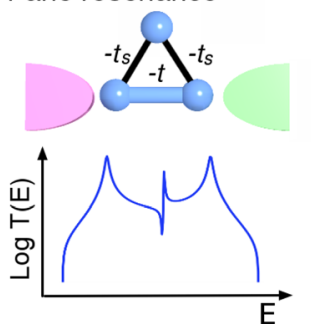

(b) Anti-resonance

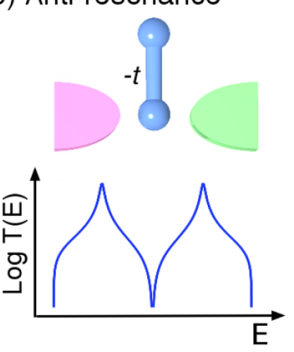

(d) Resonance

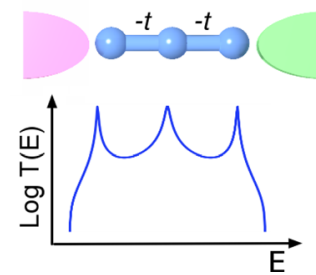

FIG. 1. Schematic picture of the four types of junctions considered in this work: (a) 2-sites, (b) T-shaped, (c) cyclic, and (d) odd-membered molecular junctions. Left (right) electrodes are shown in red (green). The typical transmission function is shown beneath each model. schematic picture of the studied systems connected between electrodes. The electronic structure of the molecular junctions is represented by a tight-binding Hamiltonian; $H=H_{\mathrm{L}}$ $+V_{\mathrm{L}}+H_{\mathrm{M}}+V_{\mathrm{R}}+H_{\mathrm{R}}$, where $H_{\mathrm{L} / \mathrm{R}}$ and $H_{\mathrm{M}}$ represent the left/right electrode and the central molecule, respectively. $V_{\mathrm{L} / \mathrm{R}}$ defines the coupling between the left/right electrodes and the molecule. The electronic propagator for the coupled system is represented by a retarded Green's function defined as $G^{\mathrm{R}}(E)=\left[(E+i \eta) I-H_{\mathrm{M}}-\Sigma_{\mathrm{L}}-\Sigma_{\mathrm{R}}\right]^{-1}$, where i $\eta$ and $\Sigma_{\mathrm{L} / \mathrm{R}}$ are an infinitesimal imaginary value and the self-energy elements which include the influence of the electrodes, respectively. The conductance of a junction at low bias and coherent regime is then obtained via the Fisher-Lee relation: $G=\frac{2 e^{2}}{h} \operatorname{Tr}\left[G^{\mathrm{R}} \Gamma_{\mathrm{L}} G^{\mathrm{A}} \Gamma_{\mathrm{R}}\right]$ where $\Gamma_{\mathrm{L} / \mathrm{R}}$ represents the broadening function given by $\Gamma_{\mathrm{L} / \mathrm{R}}(E)=i\left[\Sigma_{\mathrm{L} / \mathrm{R}}-\Sigma_{\mathrm{L} / \mathrm{R}}^{\dagger}\right]$. We used an analytic Newns-Anderson (NA) model for the electrodes, whose self-energies are given by $\Sigma_{\mathrm{L} / \mathrm{R}}(E)$ $=V_{\mathrm{L} / \mathrm{R}} g_{\mathrm{L} / \mathrm{R}}(E) V_{\mathrm{L} / \mathrm{R}}^{\dagger}$, where $g_{\mathrm{L} / \mathrm{R}}(E)$ is the surface Green's function defined as $g_{\mathrm{L} / \mathrm{R}}(E)=i \exp (i k a) / t_{\mathrm{L} / \mathrm{R}} \cdot{ }^{27,48,72-74} t_{\mathrm{L} / \mathrm{R}}$ is the nearest-neighboring (NN) transfer integral in the left/ right electrodes. We normalized all energies in $t$ and set all on-site energies to zero for simplicity. The NN coupling in the contacts are set to $t_{\mathrm{L} / \mathrm{R}}=-t$. Normally, the tight-binding parameters for organic molecules are given in units of $\mathrm{eV}$, e.g., $t=2.66 \mathrm{eV}$ for carbon-based $\pi$-electron systems. Note that the on-site energies do not always mean the energies of atomic orbitals, but could mean the eigenenergies of the fragment molecular orbitals or quantum dots.

Using nonequilibrium thermodynamics, ${ }^{75}$ the Seebeck coefficients, $S(T)$ are calculated by

$$
S(T)=(-1 / e T)\left(L_{1} / L_{0}\right),
$$

where $T$ is an average temperature, the expression $L_{n}(T)$ $=\int d E\left(E-E_{F}\right)^{n}[-\partial f(E, T) / \partial E] T_{\mathrm{el}}(E)$ are the relevant Onsager coefficients in terms of the electronic transmission function $T_{\mathrm{el}}(E)$ given by the Fisher-Lee relation shown above, and $f(E, T)$ is the Fermi function. $E_{F}$ is the Fermi energy.

\section{RESULT AND DISCUSSION}

First of all, we examined how topology affects the transmission functions and the DOS using the four types of junctions in Fig. 1. The transfer integrals are set as follows. In Fig. 1, the intra-molecular coupling are set to $-t$. In Fig. 1 (c), the coupling strengths, $t_{s}$, for two bonds between the side group and the conductor is set to $t_{s}=0.2 t$. All of the coupling strengths between the molecules and contacts are set to $V_{\mathrm{L} / \mathrm{R}}=-0.2 t$ in this study. We set the Fermi energy to $E_{\mathrm{F}}=0.0 t$. Here the role of the contacts is just to control the width of the resonances, hence different value of the coupling strength $V_{\mathrm{L} / \mathrm{R}}$ does not affect the trend of the results (for the detail, see Fig. 9 in Appendix A).

Figure 2 shows the transmissions and DOS of the four junctions in Fig. 1. The DOS of the system having two sites in Figs. 1(a) and 1(b) have the same appearance, while the system in Fig. 1(b) shows a negative peak (anti-resonance) at the Fermi energy in the transmission spectra between the resonant peaks. This anti-resonance is caused by the 

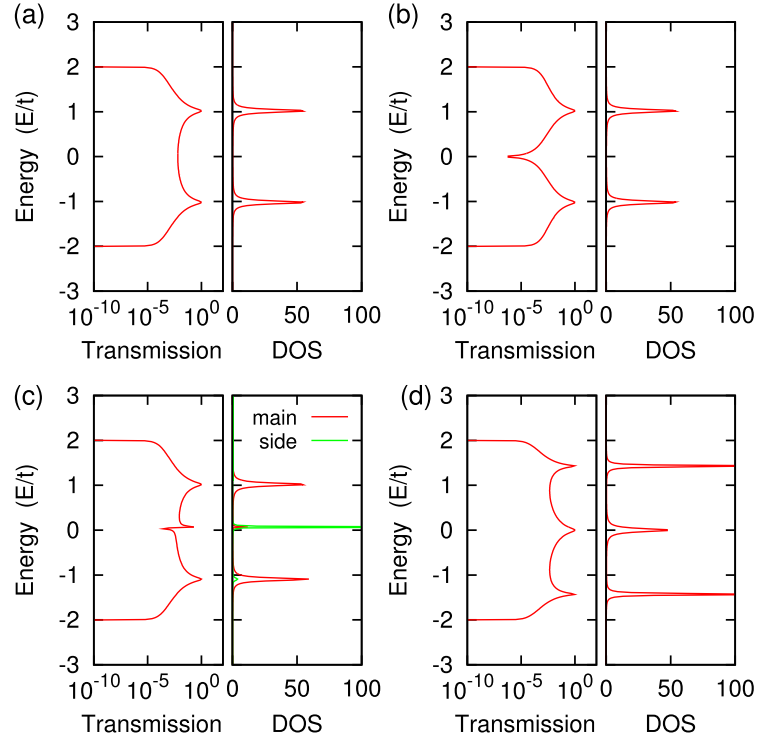

FIG. 2. Transmission functions and DOS of the four types of molecular junctions shown in Fig. 1.

cancelation of terms which describes the propagation of waves through the systems. The detailed mechanism underlying the appearance of the anti-resonance is described in Ref. 24, 27, 28, 31, 36, 38, and 41.

The switching of the resonance at $E=0.0 t$ in Figs. 2(a) and $2(\mathrm{~d})$ is commonly known as the odd-even effect. ${ }^{48}$ In general, a linear chain having odd-numbered sites tends to have resonance at the mid-gap. Interestingly, despite the symmetric topological structure of the system, the system with cyclic topology in Fig. 1(c) shows an asymmetric transmission curve around the Fermi energy (see Fig. 2(c)). This is known as the Fano resonance. ${ }^{30,76}$ This is due to the interference between the localized state from the weakly coupled side-adsorbate (see the sharp local DOS peak originating from side-attached adsorbate near the $E=0.0 t$ of Fig. 2(c)) and the tunneling state. Note that the cyclic toy model in Fig. 1(c) can be applied to realistic molecular structures as discussed in the previous section (see also Appendix B). The critical difference between the anti-resonance and the Fano resonance is that in general the anti-resonance does not require a resonant state at the position of negative peak, while the Fano-resonance requires a weakly coupled localized state near the position of the Fano peak.

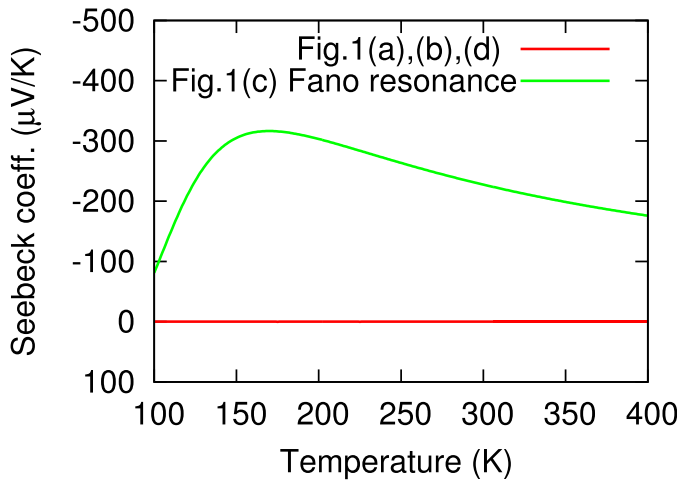

FIG. 3. Thermoelectric properties (Seebeck coefficients) of four types of junctions in Fig. 1: All on-site energies are set to zero. For the transfer integrals, $t=1.0 \mathrm{eV}$ is assumed. All systems presented no thermoelectricity except for the cyclic topology in Fig. 1(c).

Since the asymmetry or dips in transmission spectra are expected to show high thermoelectric efficiency, ${ }^{12,36,40,77}$ we estimated the Seebeck coefficients of the systems in Fig. 1. The method to calculate the Seebeck coefficient ${ }^{78-81}$ is briefly summarized above. In this work, we do not assume concrete atomistic structures, thus the vibrational contribution to thermoelectricity is not considered. Therefore, we do not estimate the figure of merit. Figure 3 shows the calculated Seebeck coefficients of the four systems in Fig. 1. Under the condition of zero on-site energies with the holeelectron symmetry, only the system with cyclic topology in Fig. 1(c) show a finite value. This feature can be exploited for the detection of cyclic topological connectivity.

The cyclic topology in Fig. 1(c) has been often used for the model of AB-rings in quantum dots in the field of mesoscopic physics. ${ }^{6,30,82}$ Due to the dephasing or an external field such as a magnetic field, the phase of the waves propagating though the different pathways in the cyclic molecule could be shifted. This could cause QI. ${ }^{15}$ In order to investigate the phase-shift effect on transport, we calculated transmission spectra and the Seebeck coefficients of the system Fig. 1(c) with different phase shifts. In Fig. 1(c), one of the bonds connecting the side adsorbate to the central molecule is changed to $t^{\prime}=t_{s} e^{i \theta}$. Fig. 4 presents the phase-shift dependence of transmission and thermoelectric properties. The phase shift weakens the Fano peaks up to $\theta=\pi / 2$, while the Fano peaks recovers over the range $\theta=\pi / 2$, finally it flips
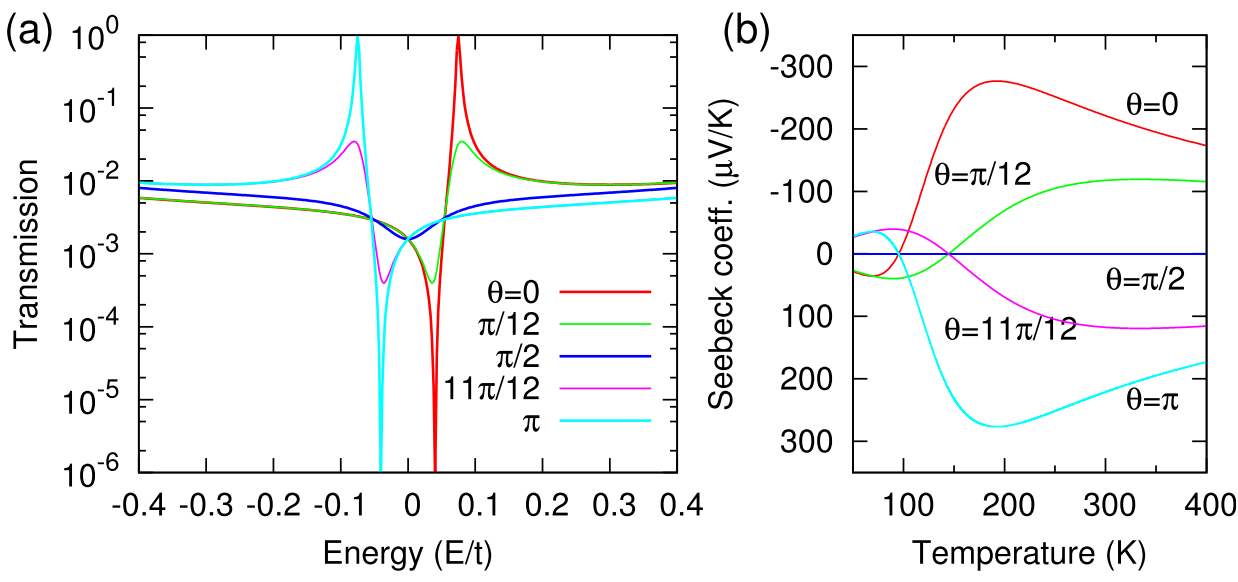

FIG. 4. Phase-shift dependence of (a) transmission profile and (b) the Seebeck coefficient for the molecular systems with cyclic topology in Fig. 1(c). In panel (b), $t=1.0 \mathrm{eV}$ is assumed. The phase of one of the transfer integrals for side-attached bonds is shifted, $t^{\prime}=t_{s} e^{i \theta}$. Interestingly, the shape of Fano resonance flips horizontally when phase is shifted for $\theta=\pi$. In this case, the sign of Seebeck coefficient also changed from negative to positive. 
horizontally at $\theta=\pi$. The sign of the thermoelectricity also changes with this transition (Fig. 4(b)). In other words, it is possible to evaluate the phase-shift from the analysis of the $S$ v.s. $T$ plot.

It is recognized that side functional groups play an essential role in QI effect. In T-shaped molecular junctions, by modulating the dihedral angle of side-attached group it is possible to change the position of the Fano resonance. ${ }^{31,36}$ When the rotational angle of the side group is large the electronic coupling between the main molecule and functional group can be reduced. In order to investigate the influence of the coupling of the side group to the transmission profile and also to the thermoelectric property, we calculated transmission spectra and Seebeck coefficients with different strengths of the side coupling. Fig. 5 shows the coupling strength dependence of (a) the transmission and (b) the thermoelectric properties of the junction in Fig. 1(c). When the coupling strength of the adsorbate in Fig. 1(c) is enhanced, the position of the Fano resonance shifts to higher energy. Then, the minimum of the $S$ vs. $T$ plot also shifts to higher temperature. Thus, it is possible to estimate the coupling strength of adsorbates from the minimum position of the $S$ vs. $T$ plot (see also Fig. 7). In the strong coupling limit, $t_{s}=t$, the Fano resonance is buried down to the normal resonance at $E=t$. Therefore, the side-attached molecules having cyclic topology with strong bindings such as cyclic propane and epoxide cannot be expected to show a Fano resonance.

Next, we examined how the thermoelectric properties of the QI-employed transport characteristics (Figs. 1(b) and
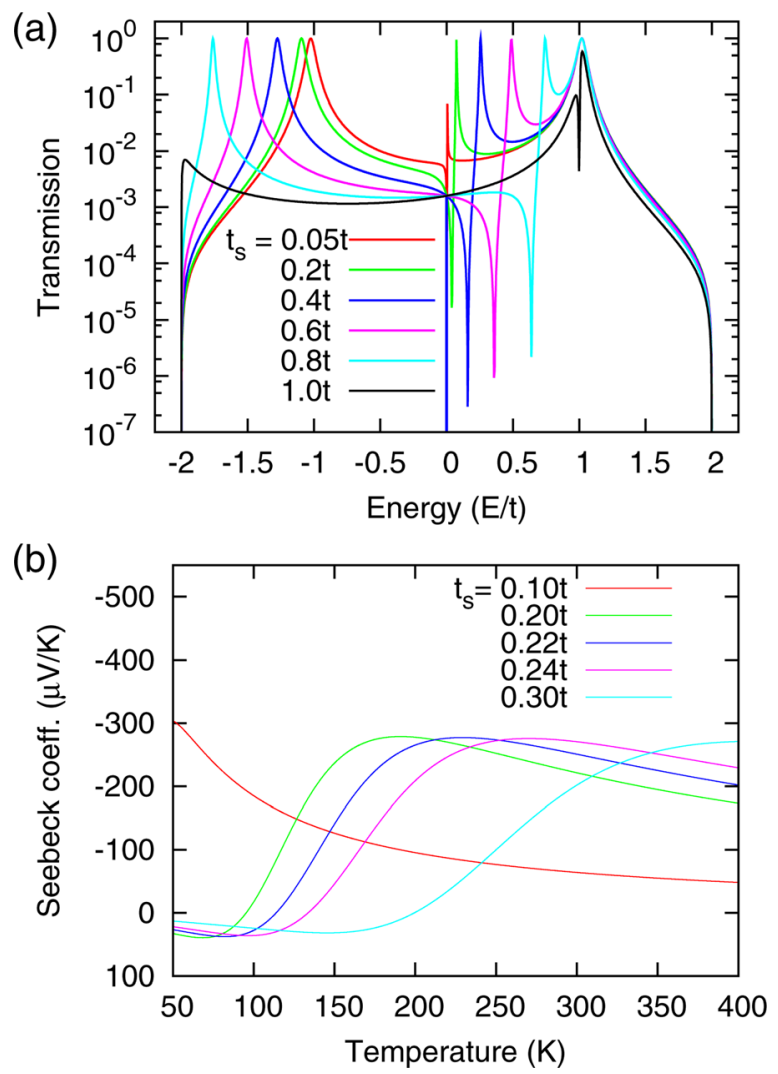

FIG. 5. Coupling strength dependence of (a) transmission profile and (b) Seebeck coefficient for the cyclic systems in Fig. 1(c). In panel (b), $t=1.0 \mathrm{eV}$ is assumed. 1(c)) depend on the electronic parameters and their topologies. We calculated the Seebeck coefficient as a function of the position of Fermi energy and temperature for the Fano resonances in Fig. 1(c) and anti-resonances in Fig. 1(b). Figures 6 and 7 shows the surface plot of Seebeck coefficient as a function of temperature, the position of the Fermi energy, and the coupling strength between a side atom and a main chain. This result suggests that by measuring thermoelectricity it is possible to infer the type of quantum interference and the topological connectivity of the molecular systems.

The transport properties in molecular systems are often affected by the disorder due to the impurity or the fluctuation of molecular structures, ${ }^{83,84}$ thus the effect of disorder on transport properties and also on the thermoelectric properties need to be assessed for the thermoelectric applications of the QI-employed molecular devices. To take this effect into account, we introduced a random shift to each of the on-site energies in the molecular region and calculated the transmission spectra and their thermoelectric properties. The transfer integrals are set to constant. The random shift of on-site energies is set to $[-W, W]$, where $W=k_{B} T$. We took 1000 samples for each toy model and analyzed the Seebeck coefficients.

Figure 8 presents the histograms of the calculated Seebeck coefficients for two different topologies, antiresonance in Fig. 1(b) and Fano resonance in Fig. 1(c), with different temperatures. The histogram for the anti-resonance in Fig. 8(a) shows symmetric distributions and the

(a)

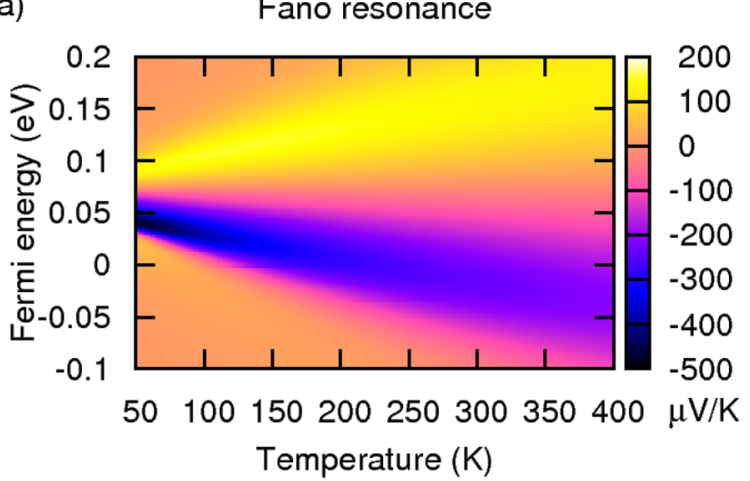

(b)

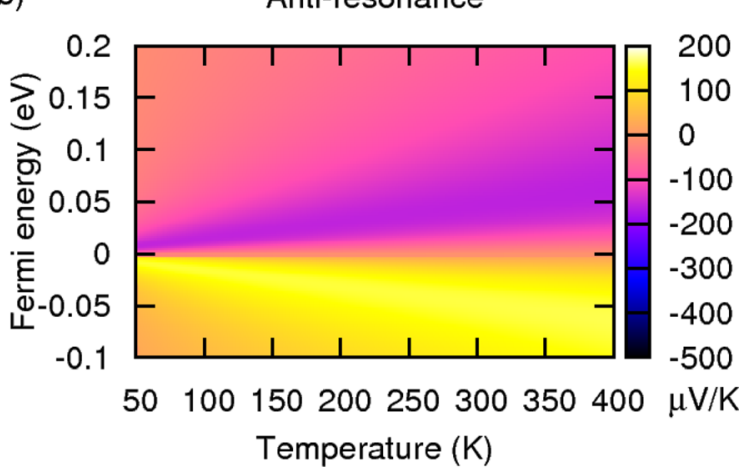

FIG. 6. Surface plot of Seebeck coefficient as a function of temperature and the position of Fermi energy for Fano resonance and anti-resonance. $t=1.0 \mathrm{eV}$ is assumed for transfer integrals. The thermoelectricity in Fano resonance is higher than that of anti-resonance. 


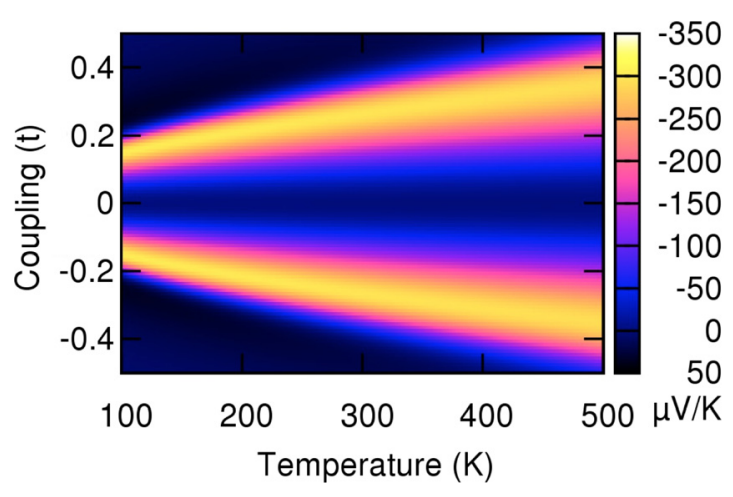

FIG. 7. Surface plot of Seebeck coefficient of Fano resonance in Fig. 1(c) as a function of temperature and the coupling strength between a side atom and a main chain.

distributions are insensitive to the temperature. In addition, the average center of the Seebeck coefficients in each temperature is close to zero, fitting well to the static analysis (see red curve in Fig. 3). On the other hand, the histogram for the Fano resonance in Fig. 8(b) shows the high and nonzero values in each temperature and in time-average. The profile of the static analysis (see green curve in Fig. 3(a)) is preserved even in the presence of the disorder of on-site energies. Therefore, the cyclic topology in Fig. 1(c) yielding
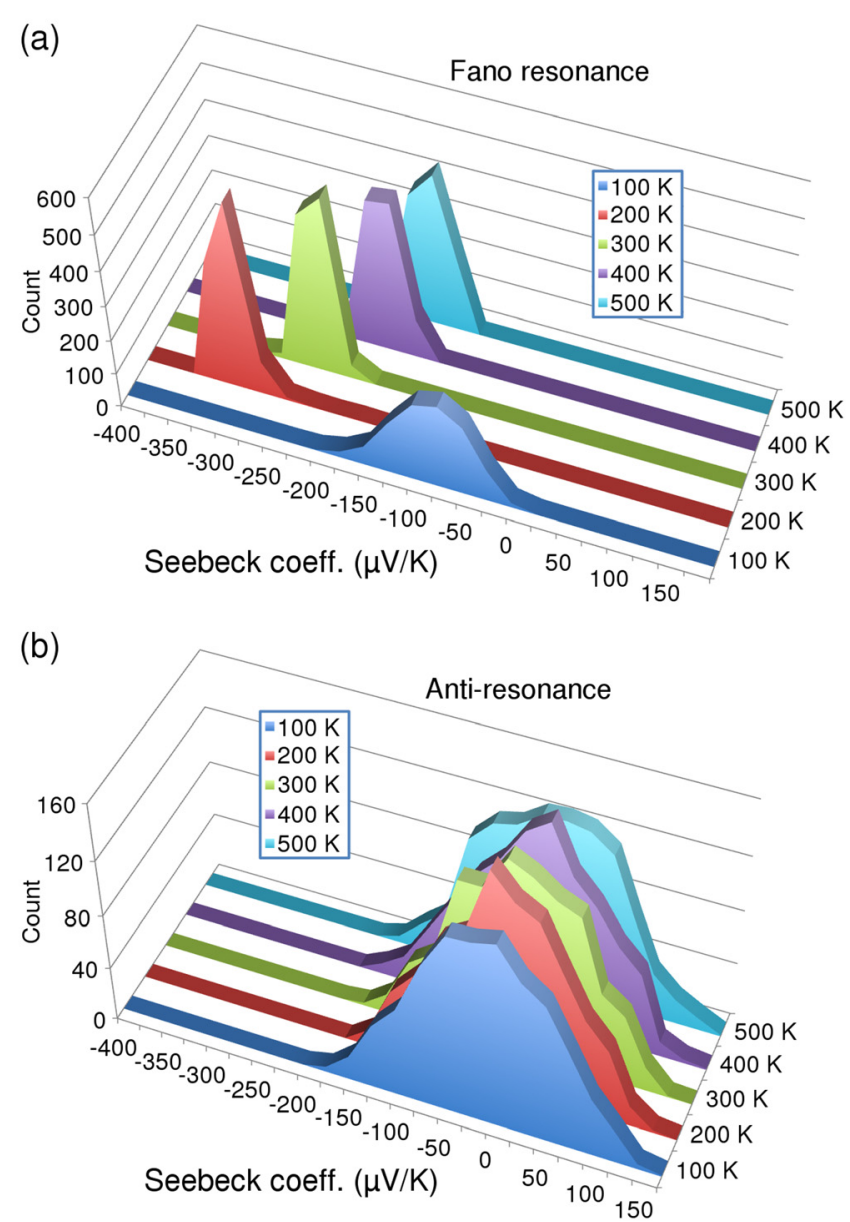

FIG. 8. Histogram of the Seebeck coefficients for (a) Fano resonance and (b) anti-resonance under thermal fluctuation of on-site energies. $t=1.0 \mathrm{eV}$ is assumed for transfer integrals in the toy models.
Fano resonance may be promising for thermoelectric applications. Although the averaged value of the Seebeck coefficients of the anti-resonance in Fig. 8(b) is lower than that of the cyclic topology, it has a merit for getting stable thermoelectric current since the Seebeck coefficient is weakly related to the temperature.

\section{CONCLUSION}

In summary, we have modeled four types of molecular junctions using a toy model and calculated the transmission function and DOS in order to investigate the relationship between the line shape of the transmission spectra and topology of the molecules. The QI features in the transmission spectra such as Fano and anti-resonance could be realized using the simple toy models having only two or three sites where all on-site energies are identical. We have also calculated the thermoelectric properties of the model systems systematically. Even under the fluctuation of the on-site energies the finite thermoelectric properties are preserved for the Fano resonance, thus cyclic configuration is promising for thermoelectric applications. This toy model analysis will help for understanding the essential physics of the interference phenomena present in t-shaped or cyclic molecular wires.

\section{ACKNOWLEDGMENTS}

We wish to acknowledge financial support from the European project Synaptic Molecular Networks for Bioinspired Information Processing (SYMONE) under Contract No. 318597. We also acknowledge the support by the German Research Foundation (DFG) within the Cluster of Excellence "Center for Advancing Electronics Dresden" (cfAED), and the European Union (European SocialFund). H.S. acknowledges support from Bilim Akademisi-The Science Academy, Turkey under the BAGEP program, support from TUBITAK-BIDEB (113C032), and TUBITAKULAKBIM High Performance and Grid Computing Center (TRUBA Resources). This work was also partially supported by the EU within the project Molecular Architectures for QCA-inspired Boolean Networks (MlArNet, Project No. 318516). We acknowledge the Center for Information Services and High Performance Computing (ZIH) at the Dresden University of Technology for computational resources.

\section{APPENDIX A: INFLUENCE OF THE COUPLING STRENGTHS $V_{L / R}$ TO THE TRANSMISSION SPECTRA AND THERMOELECTRIC PROPERTIES}

Here, we show how the coupling strengths $V_{\mathrm{L} / \mathrm{R}}$ affect the transmission spectra and thermoelectric properties in Fig. 1. Figure 9 presents the transmission spectra and DOS with stronger and weaker coupling strengths. We can see that the resonance are broadened (sharpened) in the stronger (weaker) coupling with contacts and that the shapes of the spectra are remained independent to the coupling strengths $V_{\mathrm{L} / \mathrm{R}}$. Figure 10 shows the Seebeck coefficients of the systems in Fig. 1 with stronger and weaker coupling strengths, $V_{\mathrm{L} / \mathrm{R}}$. The peak value of the Seebeck coefficients in the cyclic 

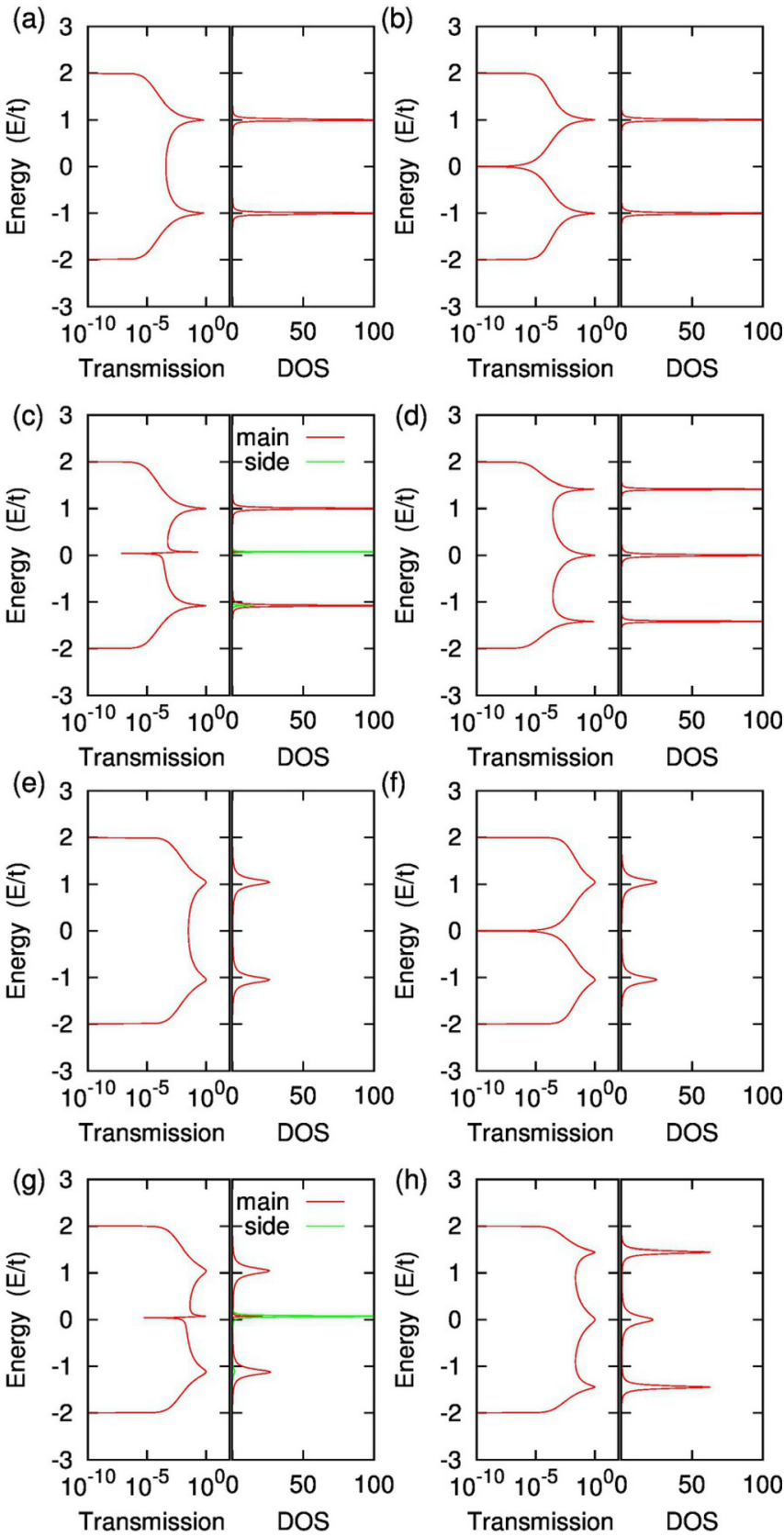

FIG. 9. Transmission functions and DOS of the four types of molecular junctions shown in Fig. 1 with (a)-(d) weaker coupling strengths $\left(V_{\mathrm{L} / \mathrm{R}}\right.$ $=-0.1 t)$ and with $(\mathrm{e})-(\mathrm{h})$ stronger coupling strengths $\left(V_{\mathrm{L} / \mathrm{R}}=-0.3 t\right)$.

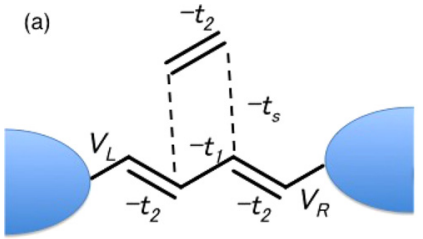

(b)
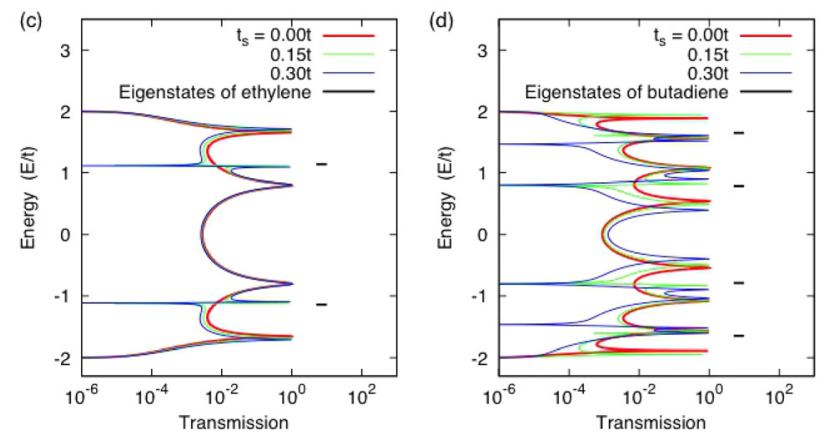

FIG. 11. Demonstration of cyclic configuration having Fano resonance in transmission spectra in molecular systems: (a) and (b) two types of $\pi$-electron systems within Hückel model. (c) and (d) Transmission functions of the molecular systems in panel (a) and (b) with different coupling strengths of $t_{s}$. We can see that the Fano resonance are created at the energies of eigenenegies of the isolated unit and that the Fano resonance gets sharper in the weaker coupling strength of $t_{s}$.

configuration is enhanced in the case of the weaker coupling strengths of $V_{\mathrm{L} / \mathrm{R}}$ (see green plots in Fig. 10(a)), while the peak value in the case of the weaker coupling in Fig. 10(a) is suppressed. This suppression is due to the broadening of the Fano resonance in the cyclic configuration as shown in Fig. $9(\mathrm{~g})$. Even with stronger or weaker coupling strengths of $V_{\mathrm{L} / \mathrm{R}}$, the trend that only the system with cyclic topology shows a finite value of the Seebeck coefficients does not change.

\section{APPENDIX B: DEMONSTRATION OF CYCLIC CONFIGURATIONS EXHIBITING FANO RESONANCE IN TRANSMISSION SPECTRA IN ORGANIC MOLECULAR SYSTEMS}

Here, we demonstrate the cyclic configurations having Fano resonance in their transmissions spectra using realistic molecular systems within Hückel basis. As target systems, we consider two types of $\pi$-electron systems in Figs. 11(a) and 11(b) where two ethylene or 1,3-butadiene units are covalently connected but another unit is stacked from top with weak inter-molecular interaction $t_{s}$. Transfer integrals for double bonds and single bonds are, respectively, set as
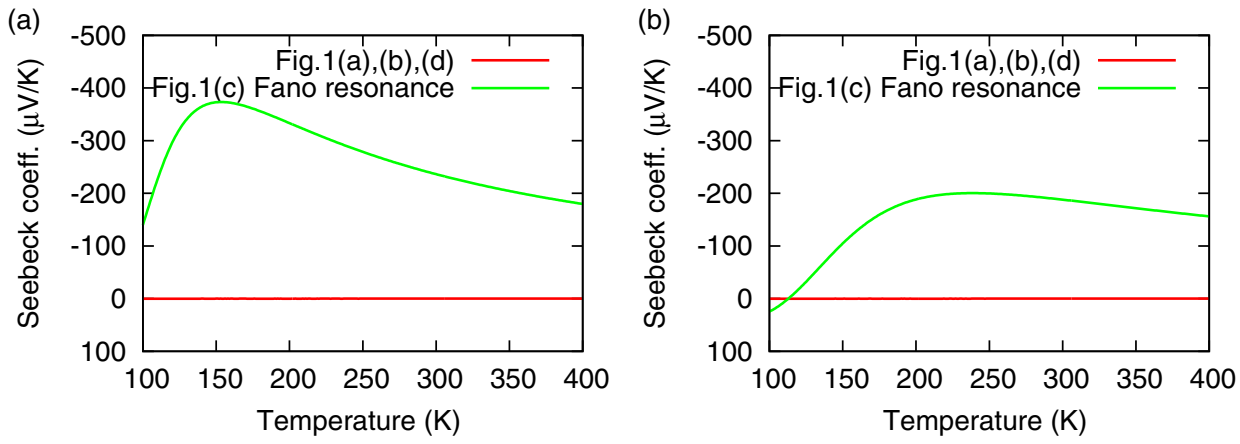

FIG. 10. Thermoelectric properties (Seebeck coefficients) of four types of junctions in Fig. 1 with (a) weaker coupling strengths $\left(V_{\mathrm{L} / \mathrm{R}}=-0.1 t\right)$ and with (b) stronger coupling strengths $\left(V_{\mathrm{L} / \mathrm{R}}=-0.3 t\right)$ : All on-site energies are set to zero. For the transfer integrals, $t=1.0 \mathrm{eV}$ is assumed. All systems presented no thermoelectricity except for the cyclic topology in Fig. 1(c). The Seebeck coefficient for the stronger couplings in panel (b) is weakened due to the broadening of the resonances. 
$-t_{2}=1.141 t$ and $-t_{1}=0.859 t$, such that the ratio between them is the same as in the literature $\left(t_{2} / t_{1}=3.65 \mathrm{eV} /\right.$ $2.75 \mathrm{eV}=1.33){ }^{85,86}$ If we focus on an eigenenergy of the fragment molecular orbital of an ethylene unit, the molecular system in Fig. 11(a) can be regarded as the cyclic configuration in Fig. 1(c). The molecular system in Fig. 11(b) can be also seen as cyclic configuration likewise if the 1,3-budadiene is considered as a unit of the system. We set the coupling strengths at the left/right contacts as $V_{\mathrm{L} / \mathrm{R}}=-0.2 t$. The left and right contacts are described with Newns-Anderson model and the parameters for the contacts are the same as Fig. 2. Figures 11(c) and 11(d) present the transmission spectra for the molecular systems in Figs. 11(a) and 11(b), respectively. As expected from the toy model analysis in Fig. 2(c), we can see that the molecular system in Fig. 11(a) (Fig. 11(b)) shows Fano resonance in its transmission spectra at the eigenenergies of an ethylene (1,3-butadiene) unit shown as black bars in Fig. 11(c). As discussed previously in Fig. 9, the role of the coupling constants $V_{\mathrm{L} / \mathrm{R}}$ is just to change the width of the resonance, thus this does not affect the shape of the transmission spectra.

${ }^{1}$ J. Barański and T. Domański, Phys. Rev. B 84, 195424 (2011).

${ }^{2}$ J. L. D'Amato, H. M. Pastawski, and J. F. Weisz, Phys. Rev. B 39, 3554 (1989).

${ }^{3}$ L. E. F. Foa Torres, H. M. Pastawski, and E. Medina, Europhys. Lett. 73, 164 (2006).

${ }^{4}$ E. Akkermans and G. Montambaux, Mesoscopic Physics of Electrons and Photons, 1st ed. (Cambridge University Press, 2007).

${ }^{5}$ P. Trocha and J. Barnaś, J. Phys.: Condens. Matter 20, 125220 (2008).

${ }^{6}$ A. E. Miroshnichenko, S. Flach, and Y. S. Kivshar, Rev. Mod. Phys. 82, 2257 (2010).

${ }^{7}$ P. Trocha, J. Phys. Condens. Matter 24, 055303 (2012).

${ }^{8}$ P. Trocha and J. Barnaś, Phys. Rev. B 78, 075424 (2008).

${ }^{9}$ M. L. Ladrón de Guevara, F. Claro, and P. A. Orellana, Phys. Rev. B 67, 195335 (2003).

${ }^{10} \mathrm{P}$. Trocha and J. Barnaś, J. Nanosci. Nanotechnol. 10, 2489 (2010).

${ }^{11}$ K. Kang, S. Y. Cho, J.-J. Kim, and S.-C. Shin, Phys. Rev. B 63, 113304 (2001).

${ }^{12}$ P. Trocha and J. Barnaś, Phys. Rev. B 85, 085408 (2012).

${ }^{13}$ P. Trocha and J. Barnaś, Phys. Rev. B 76, 165432 (2007).

${ }^{14}$ K. Kobayashi, H. Aikawa, S. Katsumoto, and Y. Iye, Phys. Rev. Lett. 88, 256806 (2002).

${ }^{15}$ W.-J. Gong, H.-M. Wang, Y. Han, and S.-J. Zhang, Curr. Appl. Phys. 14, 34 (2014).

${ }^{16}$ K. Kobayashi, H. Aikawa, A. Sano, S. Katsumoto, and Y. Iye, Phys. Rev. B 70, 035319 (2004).

${ }^{17}$ X. Yong-Jian and H. Zhou-Bo, Chin. Phys. Lett. 21, 1802 (2004).

${ }^{18}$ B. R. Bułka and P. Stefański, Phys. Rev. Lett. 86, 5128 (2001).

${ }^{19}$ L. E. Calvet, J. P. Snyder, and W. Wernsdorfer, Phys. Rev. B 83, 205415 (2011).

${ }^{20}$ G.-H. Ding, C. K. Kim, and K. Nahm, Phys. Rev. B 71, 205313 (2005).

${ }^{21}$ J. Zheng, M.-J. Zhu, and F. Chi, J. Low Temp. Phys. 166, 208 (2012).

${ }^{22}$ P. A. Orellana, F. Domínguez-Adame, I. Gómez, and M. L. Ladrón de Guevara, Phys. Rev. B 67, 085321 (2003).

${ }^{23}$ P. R. Levstein, H. M. Pastawski, and J. L. D’Amato, J. Phys. Condens. Matter 2, 1781 (1990).

${ }^{24}$ M. Ratner J. Phys. Chem. 94, 4877 (1990).

${ }^{25}$ M. Kemp, A. Roitberg, V. Mujica, T. Wanta, and M. A. Ratner, J. Phys. Chem. 100, 8349 (1996).

${ }^{26}$ E. Emberly and G. Kirczenow, Phys. Rev. Lett. 81, 5205 (1998).

${ }^{27}$ E. G. Emberly and G. Kirczenow, J. Phys. Condens. Matter 11, 6911 (1999).

${ }^{28}$ T. Tada and K. Yoshizawa, ChemPhysChem 3, 1035 (2002).

${ }^{29}$ K. Walczak, Cent. Eur. J. Chem. 2, 524 (2004).

${ }^{30}$ K. Sasada and N. Hatano, Physica E 29, 609 (2005).

${ }^{31}$ T. A. Papadopoulos, I. M. Grace, and C. J. Lambert, Phys. Rev. B 74, 193306 (2006).
${ }^{32}$ G. C. Solomon, D. Q. Andrews, R. P. Van Duyne, and M. A. Ratner, J. Am. Chem. Soc. 130, 7788 (2008).

${ }^{33}$ G. C. Solomon, D. Q. Andrews, T. Hansen, R. H. Goldsmith, M. R. Wasielewski, R. P. Van Duyne, and M. A. Ratner, J. Chem. Phys. 129, 054701 (2008).

${ }^{34}$ J. Rincón, K. Hallberg, A. A. Aligia, and S. Ramasesha, Phys. Rev. Lett. 103, 266807 (2009).

${ }^{35}$ D. A. Lovey and R. H. Romero, Chem. Phys. Lett. 530, 86 (2012).

${ }^{36}$ R. Stadler and T. Markussen, J. Chem. Phys. 135, 154109 (2011).

${ }^{37}$ T. Markussen, R. Stadler, and K. S. Thygesen, Phys. Chem. Chem. Phys. 13, 14311 (2011)

${ }^{38}$ T. Markussen, R. Stadler, and K. S. Thygesen, Nano Lett. 10, 4260 (2010).

${ }^{39}$ J. P. Bergfield, G. C. Solomon, C. A. Stafford, and M. A. Ratner, Nano Lett. 11, 2759 (2011)

${ }^{40}$ J. P. Bergfield and C. A. Stafford, Nano Lett. 9, 3072 (2009).

${ }^{41}$ T. Hansen, G. C. Solomon, D. Q. Andrews, and M. A. Ratner, J. Chem. Phys. 131, 194704 (2009).

${ }^{42}$ G. C. Solomon, J. P. Bergfield, C. A. Stafford, and M. A. Ratner, Beilstein J. Nanotechnol. 2, 862 (2011).

${ }^{43}$ C. A. Stafford, D. M. Cardamone, and S. Mazumdar, Nanotechnology 18, 424014 (2007).

${ }^{44}$ S. V. Aradhya and L. Venkataraman, Nat. Nanotechnol. 8, 399 (2013).

${ }^{45}$ L. A. Zotti, E. Leary, M. Soriano, J. C. Cuevas, and J. J. Palacios, J. Am. Chem. Soc. 135, 2052 (2013).

${ }^{46}$ A. A. Kocherzhenko, F. C. Grozema, and L. D. A. Siebbeles, Phys. Chem. Chem. Phys. 13, 2096 (2011).

${ }^{47}$ T. Markussen, J. Chem. Phys. 139, 244101 (2013).

${ }^{48}$ D. Nozaki, H. M. Pastawski, and G. Cuniberti, New J. Phys. 12, 063004 (2010).

${ }^{49}$ D. Nozaki, Y. Girard, and K. Yoshizawa, J. Phys. Chem. C 112, 17408 (2008).

${ }^{50}$ C. R. Arroyo, S. Tarkuc, R. Frisenda, J. S. Seldenthuis, C. H. M. Woerde, R. Eelkema, F. C. Grozema, and H. S. J. van der Zant, Angew. Chem. Int. Ed. 125, 3234 (2013).

${ }^{51}$ C. Arroyo, R. Frisenda, K. Moth-Poulsen, J. S. Seldenthuis, T. Bjørnholm, and H. S. J. van der Zant, Nanoscale Res. Lett. 8, 234 (2013).

${ }^{52}$ P. Sautet and C. Joachim, Chem. Phys. Lett. 153, 511 (1988).

${ }^{53}$ D. Nozaki, H. Sevinçli, S. M. Avdoshenko, R. Gutierrez, and G. Cuniberti, Phys. Chem. Chem. Phys. 15, 13951 (2013).

${ }^{54}$ S.-H. Ke, W. Yang, and H. U. Baranger, Nano Lett. 8, 3257 (2008).

${ }^{55}$ V. Rabache, J. Chaste, P. Petit, M. L. Della Rocca, P. Martin, J.-C. Lacroix, R. L. McCreery, and P. Lafarge, J. Am. Chem. Soc. 135, 10218 (2013).

${ }^{56}$ T. Markussen, J. Schiötz, and K. S. Thygesen, J. Chem. Phys. 132, 224104 (2010).

${ }^{57}$ R. Stadler, M. Forshaw, and C. Joachim, Nanotechnology 14, 138 (2003).

${ }^{58}$ R. Stadler, S. Ami, C. Joachim, and M. Forshaw, Nanotechnology 15, S115 (2004).

${ }^{59}$ R. Stadler, Phys. Rev. B 80, 125401 (2009).

${ }^{60}$ R. Stadler, K. S. Thygesen, and K. W. Jacobsen, Nanotechnology 16, S155 (2005).

${ }^{61}$ R. Stadler, "Quantum interference effects in electron transport: How to select suitable molecules for logic gates and thermoelectric devices," Architecture and Design of Molecule Logic Gates and Atom Circuits, Advances in Atom and Single Molecule Machines (Springer-Verlag, Berlin, Heidelberg, 2013), p. 25.

${ }^{62} \mathrm{H}$. Valkenier, C. M. Guédon, T. Markussen, K. S. Thygesen, S. J. van der Molen, and J. C. Hummelen, Phys. Chem. Chem. Phys. 16, 653 (2014).

${ }^{63}$ C. M. Guédon, H. Valkenier, T. Markussen, K. S. Thygesen, J. C. Hummelen, and S. J. van der Molen, Nat. Nanotechnol. 7, 305 (2012).

${ }^{64}$ W. Ren, J. Wang, Z. Ma, and H. Guo, Phys. Rev. B 72, 035456 (2005).

${ }^{65}$ S. Hershfield, K. A. Muttalib, and B. J. Nartowt, Phys. Rev. B 88, 085426 (2013).

${ }^{66}$ M. Ernzerhof, M. Zhuang, and P. Rocheleau, J. Chem. Phys. 123, 134704 (2005).

${ }^{67}$ Y. Okuno and T. Ozaki, J. Phys. Chem. C 117, 100 (2013).

${ }^{68}$ S. Ballmann, R. Härtle, P. B. Coto, M. Elbing, M. Mayor, M. R. Bryce, M. Thoss, and H. B. Weber, Phys. Rev. Lett. 109, 056801 (2012).

${ }^{69}$ T. Markussen and K. S. Thygesen, Phys. Rev. B 89, 085420 (2014).

${ }^{70}$ S. Datta, Electronic Transport in Mesoscopic Systems (Cambridge University Press, Cambridge, 1995).

${ }^{71} \mathrm{M}$. Lundstrom, Fundamentals of Carrier Transport, 2nd ed. (Cambridge University Press, Cambridge, 2009). 
${ }^{72}$ S. Datta, Quantum Transport: Atom to Transistor (Cambridge University Press, Cambridge, 2005).

${ }^{73}$ D. M. Newns, Phys. Rev. 178, 1123 (1969).

${ }^{74}$ P. W. Anderson, Phys. Rev. 124, 41 (1961).

${ }^{75}$ G. D. Mahan and J. O. Sofo, Proc. Natl. Acad. Sci. U.S.A. 93, 7436 (1996).

${ }^{76}$ U. Fano, Phys. Rev. 124, 1866 (1961).

${ }^{77}$ O. Karlström, H. Linke, G. Karlström, and A. Wacker, Phys. Rev. B 84 113415 (2011).

${ }^{78}$ C. M. Finch, V. M. García-Suárez, and C. J. Lambert, Phys. Rev. B 79, 033405 (2009).

${ }^{79}$ D. Nozaki, H. Sevinçli, W. Li, R. Gutiérrez, and G. Cuniberti, Phys. Rev. B 81, 235406 (2010).
${ }^{80}$ M. Leijnse, M. R. Wegewijs, and K. Flensberg, Phys. Rev. B 82, 045412 (2010).

${ }^{81}$ R. Świrkowicz, M. Wierzbicki, and J. Barnaś, Phys. Rev. B 80, 195409 (2009).

${ }^{82}$ Y. Aharonov and D. Bohm, Phys. Rev. 115, 485 (1959).

${ }^{83}$ D. Nozaki, C. Gomes da Rocha, H. M. Pastawski, and G. Cuniberti, Phys. Rev. B 85, 155327 (2012).

${ }^{84}$ H. Sevinçli, C. Sevik, T. Çağın, and G. Cuniberti, Sci. Rep. 3, 1228 (2013).

${ }^{85}$ J. Fink and G. Leising, Pys. Rev. B 34, 5320 (1986).

${ }^{86}$ A. J. Heeger, S. Kivelson, J. R. Schrieffer, and W.-P. Su, Rev. Mod. Phys. 60, 781 (1988). 